\title{
Population pharmacokinetics of vancomycin in Jordanian patients
}

\author{
Needa Zalloum ${ }^{1}$, Mohammad I Saleh ${ }^{1}$, Mohannad Al Haj ${ }^{2}$, Montaser Balbisi ${ }^{3}$, \\ Mutasim Al-Ghazawi ${ }^{1 *}$ \\ ${ }^{1}$ Department of Biopharmaceutics and Clinical Pharmacy, The University of Jordan, ${ }^{2}$ Department of Pharmacy, ${ }^{3}$ Department of \\ Internal Medicine, Jordan Hospital, Amman, Jordan
}

*For correspondence: Email: alghazam@ju.edu.jo

\begin{abstract}
Purpose: To characterize vancomycin pharmacokinetic properties in a Jordanian population and identify patients' characteristics that influence vancomycin disposition.

Method: A non-linear mixed-effects modeling was applied to evaluate vancomycin population pharmacokinetic parameters in Jordanian patients using NONMEM software. Vancomycin concentrations were obtained retrospectively from patients' medical records. Demographic, clinical, medication-related, and medical history data were collected and examined as potential predictors of vancomycin disposition.

Results: A total of 164 plasma vancomycin measurements from 110 patients, including neonates, older children and adults were collected. Vancomycin pharmacokinetics was described using a onecompartment model. Based on NONMEM objective function value, the selection of other models (e.g., two or three compartments) did not improve the performance of the pharmacokinetic model. Identified predictors of vancomycin clearance include: weight, serum creatinine, chronic renal failure, acute kidney injury and gender. Vancomycin volume of distribution was associated with weight and n-acetylcystine administration.

Conclusion: The present analysis is a preliminary step toward developing a vancomycin dosing algorithm in Jordanian population.
\end{abstract}

Keywords: Vancomycin, Population pharmacokinetics, NONMEM

\begin{abstract}
This is an Open Access article that uses a funding model which does not charge readers or their institutions for access and distributed under the terms of the Creative Commons Attribution License (http://creativecommons.org/licenses/by/4.0) and the Budapest Open Access Initiative (http://www.budapestopenaccessinitiative.org/read), which permit unrestricted use, distribution, and reproduction in any medium, provided the original work is properly credited.
\end{abstract}

Tropical Journal of Pharmaceutical Research is indexed by Science Citation Index (SciSearch), Scopus, International Pharmaceutical Abstract, Chemical Abstracts, Embase, Index Copernicus, EBSCO, African Index Medicus, JournalSeek, Journal Citation Reports/Science Edition, Directory of Open Access Journals (DOAJ), African Journal Online, Bioline International, Open-J-Gate and Pharmacy Abstracts

\section{INTRODUCTION}

Since its introduction in the late 1950s, vancomycin pharmacokinetic characteristics have been investigated extensively [1,2]. This could be attributed to the fact that vancomycin is associated with serious side effects. Elevated peak vancomycin concentrations ( $80 \mu \mathrm{g} / \mathrm{mL}$ ) have been associated with increased incidence of ototoxicity $[3,4]$. Additionally, the incidence of nephrotoxicity increases as vancomycin steadystate trough concentrations exceeds $15 \mu \mathrm{g} / \mathrm{mL}$ $[5,6]$.

These side effects can be minimized by appropriate selection of vancomycin dose and 
dosing interval. Appropriate therapeutic drug monitoring plans need to be individualized based on patient's characteristics. In order to achieve appropriate vancomycin dosing regimens and monitoring, investigation of vancomycin pharmacokinetic properties becomes necessary.

Vancomycin pharmacokinetic properties have been explored in several populations, including Malaysian [7], Japanese [8], Saudi [9], and Thai [10] patients. These studies were inconsistent in terms of population explored and analysis. For example, Lo et al described vancomycin pharmacokinetics in Malaysian neonates [7]. Other research groups explored adults [8-10] while Al-Kofide et al explored Vancomycin in a subgroup of Saudi adults, namely cancer patients. Furthermore, vancomycin pharmacokinetics was described using one compartment model in Malysian and Saudi patients compared to two compartment in Japanese and Thai patients [7-10]. Extrapolation of the findings of these studies to Jordanians is confounded by the aforementioned inconsistencies in the design and analysis of vancomycin pharmacokinetics. Hence, there is a knowledge gap in terms of vancomycin pharmacokinetics in Jordanian population. The objective of the present analysis is to characterize vancomycin pharmacokinetic properties in Jordanian population and identify patients' characteristics that influence vancomycin disposition. This will enable the development of vancomycin dosing algorithms and minimize the risk of vancomycin-related ototoxicity and nephrotoxicity.

\section{METHODS}

IRB approval to review medical records of human patients was obtained through the medical ethics committee at Jordan Hospital (dated 26 ${ }^{\text {th }}$, September 2012 with a study title of Population Pharmacokinetics of Vancomycin in Jordanian Patients). The study was conducted according to ICH-GCP guidelines that are consistent with the Declaration of Helsinki [11,12]. Routine clinical data were retrospectively collected from 110 patients who received vancomycin therapy and had at least one recorded vancomycin measurement. Patients were treated at Jordan Hospital (Amman, Jordan) between January 2011 and December 2012. The collected data were as follows: (1) demographic data (age, sex, height, and total weight); (2) clinical data (indication for vancomycin use, mechanical ventilation status, nutritional support, and laboratory data [e.g., serum creatinine; ALT and AST levels; biliurubin level; blood urea nitrogen; fluid balance; and albumin levels]); medication history (dosage regimen of vancomycin, concomitant medications, date and time of administration, sampling time of vancomycin); and (4) medical history. Several measurements of serum creatinine (and creatinine clearance) were obtained for several patients. The value of serum creatinine or creatinine clearance used in the model was obtained using linear interpolation.

A total of 164 vancomycin measurements were included in the analysis. An average of 1.5 measurements were recorded from each patient (range $1-5$ measurements/patient),. Measurement of serum vancomycin concentrations was conducted as part of therapeutic drug monitoring activity in the hospital using the fluorescence polarization immunoassay (FPIA) method with Axsym system (package insert, Axsym system, Abbot Laboratories, Abbot Park, III, USA).

\section{Pharmacokinetic analysis}

Pharmacokinetic model development and covariate screening was conducted using NONMEM 6 (ICON Development Solutions, Ellicott City, MD). FOCE estimation option with three or more significant digits was used. Models with unsuccessful minimization were excluded and were not carried forward. Additionally, model selection was based on, a significant $(p<0.05)$ decrease in objective function (>3.84), visual evaluation of goodness of fit plots. These plots include: observed concentrations against individual predicted concentrations, observed concentrations against population predicted concentrations, weighted residual(using individual and population predictions) over time), parameter estimates precision (i.e. standard error of the mean), and changes in betweensubject and residual variability.

\section{Pharmacokinetic model development}

Several pharmacokinetic models (1, 2 and 3 compartment models) were examined. A lognormal distribution was used to describe between-subject variability: $P_{i}=\theta_{k} \exp \left(\eta_{\mathrm{ki}}\right)$, where $\mathrm{Pi}$ is the estimated pharmacokinetic parameter for the $i^{\text {th }}$ subject, $\theta_{k}$ is the average population value of parameter $\mathrm{k}$, and $\eta_{\mathrm{ki}}$ is the inter-individual random effect for the $i^{\text {th }}$ subject and the $k^{\text {th }}$ parameter. Clearance (CL) and volume of distribution ( $\mathrm{Vd}$ ) were estimated as primary parameters.

The residual error of the data was assumed to be proportional to the measured concentration (proportional error). Correlations between 
estimated pharmacokinetic parameters were examined using visual inspection of estimated random effects ( $(\eta)$ of pharmacokinetic parameters.

\section{Covariate analysis}

The correlation between pharmacokinetic parameters and several potential covariates, from clinical or biological view, with was examined using covariate screening. Covariate screening was performed using forward addition with subsequent backward elimination procedure. First, vancomycin measurements was modeled using the basic structural model described (i.e., one, two or three compartments) without including patients' characteristics as covariates. Second, each patient's characteristic was included in the mixed-effects model separately. Third, patients' characteristics that resulted in a significant drop in NONMEM objective function were added simultaneously to the mixed effects model. Finally, step 3 was repeated for several iterations. In each iteration one covariate was excluded. The covariates that did not result in significant increase (compared to the model with all significant covariates) in NONMEM objective function were excluded from the final model. Significant change in NONMEM objective functions was defined, as a change of more than 3.84 is equivalent to a $p$-value of less than 0.05 . The selection of a cut-point of 3.84 was based on the previous finding that change in the objective function is equivalent to the change in twice the negative log-likelihood function. Hence, chi-square distribution (degree of freedom $=1$ ) is commonly used as an approximation of the change in NONMEM objective function $[13,14]$.

\section{Model evaluation}

Accuracy of estimated pharmacokinetic parameters was examined using bootstrap analysis. Ninety-fifth percentile confidence interval was estimated for all parameters. Resampling with replacement of the original dataset was used to generate 1000 simulated datasets. Pharmacokinetic parameters were estimated, using NONMEM, for each simulated datasets. This resulted in 1000 estimate of each pharmacokinetic parameter. These estimates were then used to calculate $95^{\text {th }}$ percentile confidence interval

The model predictive performance was examined using standardized visual predictive check (SVPC) as described by Wang [15]. One thousand datasets were simulated using the estimated pharmacokinetic parameters, estimated between-subject variability, and estimated residual variability. The simulated datasets are identical to the original dataset in terms of number of individuals, dose administered, and vancomycin sampling timepoints). NONMEM was used to simulate these datasets using the same variance model used in the final pharmacokinetic model (i.e. lognormal distribution for inter-individual variability and proportional error for residual variability). The percentile of each observation was then plotted vs. time. The percentile $\left(P_{i j}\right)$ of $\mathrm{j}^{\text {th }}$ observation for the $\mathrm{i}^{\text {th }}$ subject was calculated as in Eq 1 .

$$
P_{i j}=\frac{1}{1001}\left(1+\sum_{k=1}^{1000} \delta_{i j, k}\right)
$$

where $\delta_{i j, k}=1$ if $y_{i j}>y_{i j, k}^{\prime}$, otherwise, $\delta_{i j, k}=0, y_{i j}$ is the actual $j^{\text {th }}$ measured vancomycin concentration for the $\mathrm{i}^{\text {th }}$ subject, and $y_{i j, k}^{\prime}$ is the $\mathrm{k}^{\text {th }}$ simulated concentration corresponding to $y_{i j}$.

\section{RESULTS}

The age ranged between 14 days to 88 years with an average of 46.1 years (standard deviation, 25.6). About one third (34\%) of the explored subjects were females. The average total body weight was $63.5 \mathrm{~kg}$ (range: 1.3 to 115 $\mathrm{kg}$ ). Even though this study was retrospective, we were able to obtain medical history information from patients' medical files. Regarding the medical history, $17 \%$ presented with heart failure, $18 \%$ presented with chronic renal failure, $36 \%$ experienced septic shock during the study, and $45 \%$ developed acute kidney injury during the study.

One compartment model was selected as structural model. The addition of more compartments (i.e. distribution compartments) did not result in significant $(p<0.05)$ improvement in the model performance (according to NONMEM objective function). Between-subject variability modeled for clearance.

Identified predictors of vancomycin clearance include: weight (WT), serum creatinine, chronic renal failure (CRF), acute kidney injury (AKI), and gender. Vancomycin volume of distribution was associated with weight and n-acetylcystine administration. Equations 2 and 3 presents proposed regression equations that quantify the contribution of various patients' characteristics and pharmacokinetic parameters. Regression coefficients are summarized in Table 1. 
$\log (\mathrm{CL})=\log \left(\mathrm{Cl}_{0}\right)+\beta 1 * \log (\mathrm{WT})+\beta 2 * \mathrm{CRF}+\beta 3 * \mathrm{AKI}+\beta 4 *$ Gender (2)

where $\beta 1, \beta 2, \beta 3$, and $\beta 4$ are parameters that quantify the contribution of weight, CRF, AKI and gender on vancomycin clearance.

$\log (\mathrm{V})=\log \left(\mathrm{V}_{0}\right)+\beta 5 * \log (\mathrm{WT})+\beta 6 * \mathrm{NAC}$ ..(3)

where $\beta 5$ and $\beta 6$ are parameters that describe the influence of weight and n-acetylcystine (NAC) administration on vancomycin volume of distribution.

The goodness of fit plots are presented in Figures 1, Figure 2 and Figure 3. Population predicted concentrations (PRED) and individual predicted concentrations (IPRED) provide unbiased estimates of observed vancomycin concentrations (Figure 1). Weighted residuals for population (WRES, Figure 2) and individual (IWRES, Figure 3) were randomly scattered around 0 with no apparent trend. This finding was consistent over time. A good predictive performance of the model can be inferred from SVPC plot (Figure 3). Percentiles of observed vancomycin concentrations relative to SVPC simulated concentrations were evenly distributed between 0 and 1 . The diagnostic plots demonstrate the adequacy of the model and population parameter estimates.

\section{DISCUSSION}

A pharmacokinetic model was developed to describe the longitudinal change in vancomycin concentrations. Furthermore, several predictors of vancomycin clearance and/or volume of distribution were identified. A one-compartment model was selected. Identified predictors of vancomycin clearance include: weight, serum creatinine, chronic renal failure, acute kidney injury, and gender. Vancomycin volume of distribution was associated with weight and nacetylcystine administration.
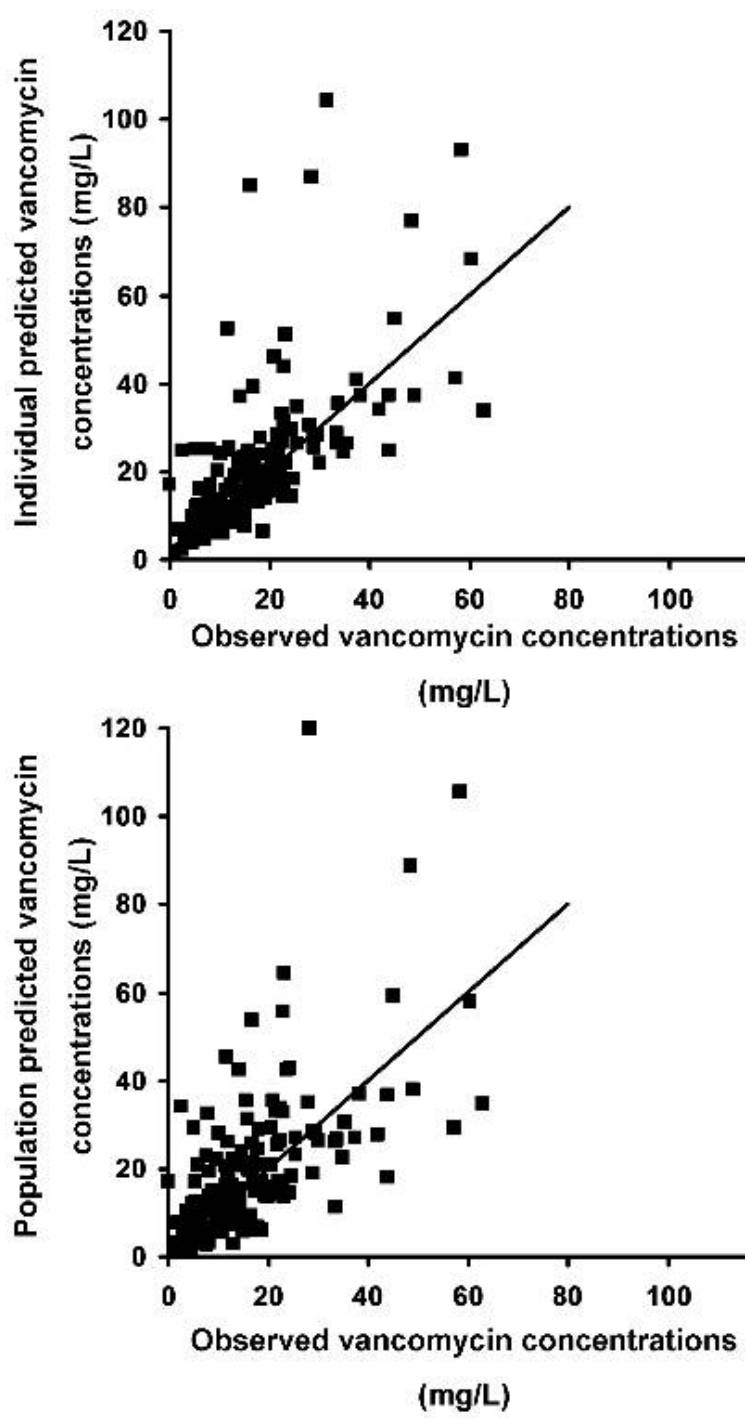

Figure 1: Observed versus individual predicted (upper) and population predicted (lower) concentrations of vancomycin

Table 1: Estimated pharmacokinetic parameters for vancomycin population pharmacokinetic model

\begin{tabular}{llc}
\hline Parameter & Description & $\begin{array}{c}\text { Typical population estimate } \\
\text { (bootstrap95\% Cl) }\end{array}$ \\
\hline $\mathrm{Cl}, \mathrm{L} / \mathrm{hr}$ & Clearance & $0.046(0.02,0.08)$ \\
$\mathrm{Cl}-\mathrm{IIV}$ & Inter individual variability in clearance values & $42.8 \%(8 \%, 56 \%)$ \\
$\mathrm{V}, \mathrm{L}$ & Volume of distribution & $0.867(0.48,3.07)$ \\
$\beta 1,1 / \mathrm{kg}$ & The influence of weight $(\mathrm{Wt})$ on clearance & $0.979(0.84,1.16)$ \\
$\beta 2, \mathrm{dL} / \mathrm{mg}$ & The influence of serum creatinine on clearance & $-0.333(-0.98,-0.31)$ \\
$\beta 3$ & The influence of chronic renal failure on clearance & $-1.960(-3.55,-0.91)$ \\
$\beta 4$ & The influence of acute kidney injury on clearance & $-0.645(-0.93,-0.03)$ \\
$\beta 5$ & The influence of gender on clearance & $0.366(0.06,0.66)$ \\
$\beta 6$ & The influence of weight on volume of distribution & $0.986(0.69,1.13)$ \\
$\beta 7$ & The influence of n-acetyl cystine on volume of distribution & $0.927(0.53,1.31)$ \\
\hline
\end{tabular}



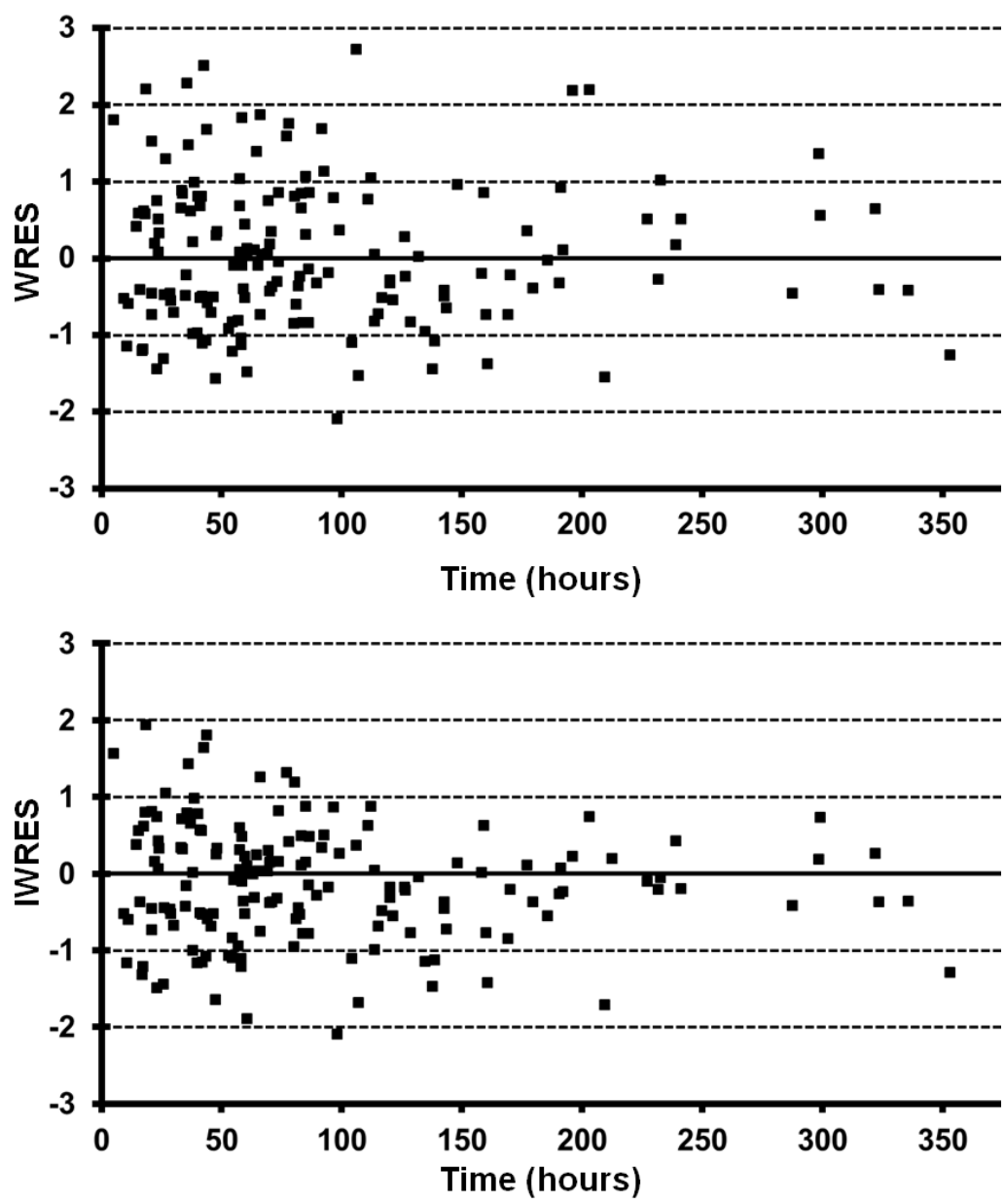

Figure 2: Population (WRES) and individual (IWRES) weighted residuals versus time

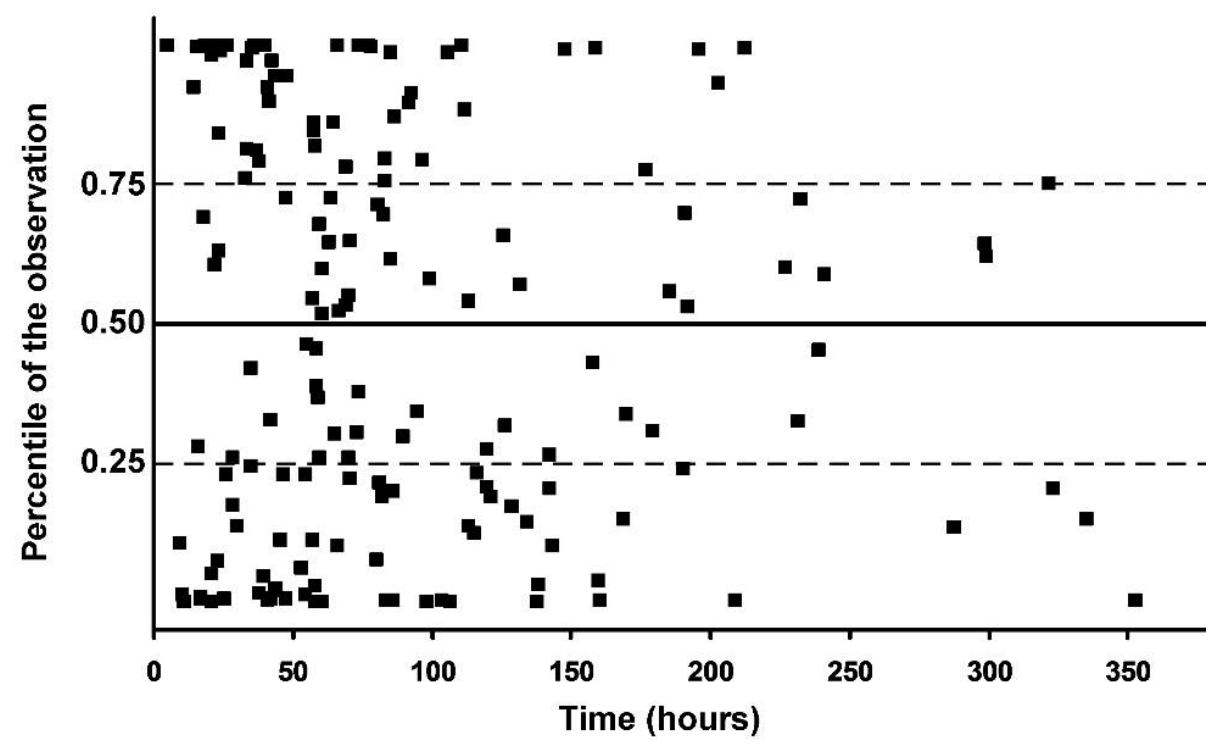

Figure 3: Standardized visual predictive check (SVPC) plot for vancomycin population pharmacokinetic model

Most of the variables identified by the present analysis are in line with previous reports. For example, several independent research groups reported that weight $[16,17]$, serum creatinine [18], acute kidney injury [19], chronic renal failure affected vancomycin clearance and/or volume of distribution.

Vancomycin is primarily ( $\geq 90 \%$ ) eliminated unchanged in the urine by glomerular filtration 
[3]. In addition of renal clearance, non-renal clearance of vancomycin is reduced in patients with acute or chronic renal failure [19-21]. Hence, it is expected for serum creatinine level to play a key role in predicting vancomycin clearance. Increased serum creatinine level is a marker of reduced kidney function and as a result decreased vancomycin clearance. Decreased vancomycin clearance is an obvious consequence of chronic renal failure [21]. Previous reports have demonstrated that vancomycin half-life is prolonged in patients with renal failure with no apparent change in the volume of distribution. Similarly, more extensive vancomycin accumulation is expected in patients with acute kidney injury [19].

Being male is associated with increased value of creatinine clearance. Gender affects vancomycin clearance as a direct consequence of its influence on creatinine clearance. In other words, adult males have higher creatinine clearance compared to adult females of the same age, weight, and serum creatinine values [22].

This is the first report of association between $\mathrm{N}$ acetylcystine administration and vancomycin pharmacokinetics. Previous study reported decreased vancomycin-associated ototoxicity risk as a result of $\mathrm{N}$-acetylcystine use [23]. Based on the present findings, a speculative explanation for the protective effect of $\mathrm{N}$ acetylcystine is that $\mathrm{N}$-acetylcystine increases the volume of distribution of vancomycin. As a result, vancomycin concentrations are expected to be lower in patients who received $\mathrm{N}$ acetylcystine. Hence, the incidence of ototoxicity is expected to be lower [24]. On the other hand, in the investigated cohort, 7 patients received $\mathrm{N}$ acetylcystine. The present finding cannot be extrapolated to a larger population without further investigation.

The present analysis offers several advantages. First, it is the first population pharmacokinetic analysis of vancomycin in Jordanian population that included a wide age range (14 days - 88 years). All patients received vancomycin as intravenous infusion. The change in pharmacokinetic parameters with age was accounted for in the model using body weight as covariate for both volume of distribution and clearance. Hence, adult patients are expected to have larger body weight compared to paediatric patient. According to the model, this will result in larger volume of distribution and clearance. Badran et al explored vancomycin pharmacokinetics in neonates admitted to the Jordan University Hospital [25]. However, they did not use population pharmacokinetics approach that has the ability of explaining variability observed among patients using patients' characteristics [26-28]. Additionally, the previously explored cohort did not include adults. Furthermore, the present methodology explicitly accounts for changing kidney function measures. This was implemented using linear interpolation of serum creatinine values. This is valuable in case where patients experience acute kidney injury during the study. In this case, using serum creatinine values at the beginning failed to account for the decreasing kidney function that was observed at later time points.

\section{Limitations of the study}

Perhaps the main limitation of the present analysis is that it is a retrospective analysis. Hence, the investigators did not control the study design. For example, dose and sampling time points selection were based on the decision of the attending physician. Selecting sampling time points and number is important in capturing more extensive pharmacokinetic characteristics such as vancomycin tissue distribution. This explains the failure in selecting a more complicated pharmacokinetic model such as two compartments model.

\section{CONCLUSION}

A population pharmacokinetic model of vancomycin in Jordanian population has been presented in this manuscript. Additionally, several patient characteristics have been identified as predictors of vancomycin pharmacokinetic characteristics. The present analysis is a preliminary step toward developing a vancomycin dosing algorithm in Jordanian population.

\section{DECLARATIONS}

\section{Acknowledgement}

The authors would like to extend their appreciation to the management and staff of Jordan Hospital for facilitating access to the patient records. Also, we would like to thank The University of Jordan represented by the Deanship of Academic Research for supporting and funding the project. This work was carried out during the sabbatical leave granted to one of the authors (Mutasim Al-Ghazawi) from University of Jordan during the academic year 2014/2015. 


\section{Conflict of interest}

No conflict of interest is associated with this work.

\section{Contribution of authors}

The authors declare that this work was done by the authors named in this article and all liabilities pertaining to claims relating to the content of this article will be borne by them. Needa Zalloum: Study design, data collection, and manuscript preparation; Mohammad I Saleh: Study design, data analysis, and manuscript preparation; Mohannad Al Haj: Study design, data collection, and manuscript preparation; Montaser Balbisi: Study design, data collection, and manuscript preparation; Mutasim Al-Ghazawi: Study design, data analysis, and manuscript preparation

\section{REFERENCES}

1. McCormick MH, McGuire JM, Pittenger GE, Pittenger $R C$, and Stark WM. Vancomycin, a new antibiotic. I. Chemical and biologic properties. Antibiot Annu 1955; 3 : 606-611.

2. Levine DP. Vancomycin: a history. Clin Infect Dis 2006; 42 Suppl 1: S5-12.

3. Kirby WM, Divelbiss CL. Vancomycin; clinical and laboratory studies. Antibiot Annu 1956: 107-117.

4. Geraci JE, Heilman FR, Nichols DR, Wellman EW, and Ross GT. Some laboratory and clinical experiences with a new antibiotic, vancomycin. Antibiot Annu 1956: 90 106.

5. Zimmermann AE, Katona BG, Plaisance KI. Association of vancomycin serum concentrations with outcomes in patients with gram-positive bacteremia. Pharmacother 1995; 15: 85-91.

6. Hidayat LK, Hsu DI, Quist R, Shriner KA, and WongBeringer A. High-dose vancomycin therapy for methicillin- esistant Staphylococcus aureus infections: efficacy and toxicity. Arch Intern Med 2006; 166: 21382144.

7. Lo YL, van Hasselt JG, Heng SC, Lim CT, Lee TC, and Charles BG. Population pharmacokinetics of vancomycin in premature Malaysian neonates: identification of predictors for dosing determination. Antimicrob Agents Chemother 2010; 54: 2626-2632.

8. Yasuhara $M$, Iga $T$, Zenda $H$, Okumura $K$, Oguma $T$, Yano $Y$, and Hori R. Population pharmacokinetics of vancomycin in Japanese adult patients. Ther Drug Monit 1998; 20: 139-148.

9. Al-Kofide H, Zaghloul I, Al-Naim L. Pharmacokinetics of vancomycin in adult cancer patients. J Oncol Pharm Pract 2010; 16: 245-250.

10. Purwonugroho TA, Chulavatnatol S, Preechagoon $Y$, Chindavijak $B$, Malathum $K$, and Bunuparadah $P$.
Population pharmacokinetics of vancomycin in Thai patients. Scientific World J 2012; 2012: 762649.

11. Association WM. Declaration of Helsinki. Ethical principles for medical research involving human subjects. http://www. wma. net/e/policy/b3. htm 2008.

12. Guideline IHT, Guideline for Good Clinical Practice. E6 (R1). 1996. 2013.

13. Takama H, Tanaka $H$, Nakashima D, Ueda $R$, and Takaue Y. Population pharmacokinetics of intravenous busulfan in patients undergoing hematopoietic stem cell transplantation. Bone Marrow Transplant 2006; 37: 345351.

14. Stroup TS, Lieberman JA, Antipsychotic trials in schizophrenia: The CATIE project. Cambridge medicine. 2010, Cambridge, UK; New York: Cambridge University Press. xvii, 312

15. Wang $D D$, Zhang S. Standardized visual predictive check versus visual predictive check for model evaluation. $J$ Clin Pharmacol 2012; 52: 39-54.

16. Marques-Minana MR, Saadeddin A, Peris JE. Population pharmacokinetic analysis of vancomycin in neonates. $A$ new proposal of initial dosage guideline. $\mathrm{Br} J$ Clin Pharmacol 2010; 70: 713-720

17. Yasuhara $M$, Iga $T$, Zenda $H$, Okumura $K$, Oguma $T$, Yano $Y$, and Hori R. Population pharmacokinetics of vancomycin in Japanese pediatric patients. Ther Drug Monit 1998; 20: 612-618.

18. Kimura $T$, Sunakawa $K$, Matsuura $N$, Kubo $H$, Shimada $S$, and Yago K. Population pharmacokinetics of arbekacin, vancomycin, and panipenem in neonates. Antimicrob Agents Chemother 2004; 48: 1159-167.

19. Macias WL, Mueller BA, Scarim SK. Vancomycin pharmacokinetics in acute renal failure: preservation of nonrenal clearance. Clin Pharmacol Ther 1991; 50: 688694.

20. Matzke GR, McGory RW, Halstenson CE, and Keane WF. Pharmacokinetics of vancomycin in patients with various degrees of renal function. Antimicrob Agents Chemother 1984; 25: 433-437.

21. Moellering RC, Jr., Krogstad DJ, Greenblatt DJ. Pharmacokinetics of vancomycin in normal subjects and in patients with reduced renal function. Rev Infect Dis 1981; 3 suppl: S230-235.

22. Cockcroft DW, Gault MH. Prediction of creatinine clearance from serum creatinine. Nephron 1976; 16: 31 41.

23. Abedi Azar S, Emami Pour M, Nakhjavani MR, Etemadi J, Zolali $\mathrm{N}$, Kyanvar $\mathrm{H}$, and Naderpoor $\mathrm{M}$. Effect of $\mathrm{N}$ acetylcysteine in the prevention of ototoxicity caused by vancomycin in the treatment of peritonitis in patients undergoing peritoneal dialysis. Int $J$ Curr Res Acad rev 2014; 2: 226-235.

24. Kirby WM, Perry DM, Bauer AW. Treatment of staphylococcal septicemia with vancomycin: report of thirty-three cases. N Engl J Med 1960; 262: 49-55.

25. Badran EF, Shamayleh A, Irshaid YM. Pharmacokinetics of vancomycin in neonates admitted to the neonatology 
unit at the Jordan University Hospital. Int J Clin Pharmacol Ther 2011; 49: 252-257.

26. Seltman HJ, Experimental Design and Analysis. 2013, Pittsburgh, PA: Carnegie Mellon University.

27. Saleh MI. Modeling longitudinal changes in buprenorphine treatment outcome for opioid dependence. Pharmacopsychiat 2014; 47: 251-258.
28. Saleh MI, Nalbant D, Widness JA, and Veng-Pedersen $P$. Population pharmacodynamic analysis of erythropoiesis in preterm infants for determining the anemia treatment potential of erythropoietin. Am J Physiol Regul Integr Comp Physiol 2013; 304: R772-781. 